\title{
MANAGING MISTRUST IN CONSTRUCTION USING DLT: A REVIEW OF USE-CASE CATEGORIES FOR TECHNICAL DESIGN DECISIONS
}

\author{
Jens J. Hunhevicz, Daniel M. Hall \\ Institute of Construction and Infrastructure Management, Chair of Innovative and Industrial Construction, \\ ETH Zurich, Zurich, Switzerland
}

\begin{abstract}
Blockchain, or more general distributed ledger technology (DLT), is seen as an opportunity to integrate digital information, management, and contracts to increase trust and collaboration within the construction industry. However, little research has analyzed and confirmed this linkage through case-studies. There seems to be a gap between use-case ideas and the technical system implementation. This paper aims to reduce this gap through a review of both DLT and its applications in construction. It proposed six use-case categories through clustering of analyzed use cases and a basic framework to match the categories to a suitable DLT design. For that, the trust level of the user group was used as a proxy for the needed fundamental properties of the system. The categories and framework can lead to a more connected and structured thinking between technological properties of DLT's and use cases in construction.
\end{abstract}

\section{Introduction}

Construction project teams often work in cross-functional, geographically distributed teams (Zolin et al. 2004) composed of complex and fragmented supply chains. The successful completion of complex projects requires the development of trust and mutual confidence between the interacting parties (Pishdad-Bozorgi and Beliveau 2016). This has been found to be a major challenge for large, complex projects that rely on the interdependent actions of numerous stakeholders (Zolin et al. 2004). Without a strong foundation of trust, it is difficult to reach consensus and information exchange in a meaningful manner (Hall et al. 2014). Mistrust leads to guarded behaviors and conflict within project teams and results in individuals pursuing and protecting their own interests instead of the benefit of the overall projects (Pishdad-Bozorgi and Beliveau 2016). Furthermore, the construction industry suffers from longitudinal fragmentation that occurs when project teams disband at the end of projects and are selected on future projects by competitive bidding. They are thus unlikely to work with the same set of partner firms on future projects. Consequently, team members lose tacit knowledge about how to work together effectively (Dubois and Gadde 2002) and organizations are unable to build long-term trusting relationships across firm boundaries. It is therefore not surprising that project sponsors and clients list a primary concern to be "trust and integrity in the construction process" (Thomsen et al. 2009).
Scholars and practitioners argue that digital transformation can help overcome challenges of mistrust and fragmentation in order to increase the overall productivity of the construction industry (Agarwal et al. 2016; Whyte and Hartmann 2017). Digital tools such as Building Information Modelling (BIM) promises potential in this regard. BIM allows designers and builders to design, visualize, and coordinate construction systems with greater efficiency through the use of three-dimensional modelling tools and processes. While helpful for individual firms, BIM provides significantly more value when it can integrate information across multiple firms and organizations in the supply chain (Papadonikolaki and Wamelink 2017). However, the use of a shared model requires again trust in the other project participants that originally created the model (Hall et al. 2014); this trust is often not found in BIM practice (Miettinen and Paavola 2014; Papadonikolaki 2018). Despite its potential, the adoption of BIM has lagged as project teams struggle with trust and liability concerns associated with sharing information on the project (Hall and Scott 2019). It seems that new technologies such as BIM are important but can only offer a partial solution to trust and liability.

The emergence of Distributed Ledger Technologies (DLT), also known as blockchain, offers an opportunity to increase trust and collaboration within the construction industry by integrating digital information with management and contracts. It can help making the construction process more efficient, transparent, and accountable between all involved participants (Penzes 2018). Past research has identified and summarized specific use cases where DLT can be applied to construction (e.g. Li et al. 2018). However, DLT is an emerging concept. Previous reviews of DLT have determined lists of single use cases but have not clustered applications into higher-order categorizations. Furthermore, past reviews of use cases lack consideration of the fundamental design decisions made for DLT's. DLT's can be chosen with different levels of decentralization according to the need of the users, which also impacts a DLT's ability to overcome issues of trust. Much existing literature in construction does not take the technical design of DLT's into consideration when reviewing its potential use cases.

This paper attempts to further refine the understanding of potential use cases for DLT in construction. To do so, we 
review nine papers that identify multiple potential use cases for DLT in construction. From these papers, we identify 22 use cases. These use cases are then clustered into six higher-order use-case categories. For each of the six use-case categories, we map the technical requirements of the DLT's onto the design decisions. The resulting framework is useful for researchers and practitioners to identify the technical design decisions required for future use-case implementations of DLT in construction.

\section{Distributed Ledger Technology}

Bitcoin as a blockchain-based crypto-currency introduced by Satoshi Nakamoto (2008) emerged as a combination of existing technologies (e.g. distributed ledgers, public-key encryption, merkle tree hashing, consensus protocols) (Tasca and Tessone 2017). Often called a disruptive technology, blockchain enables application beyond the specific case of Bitcoin as peer-to-peer money. It is possible with this new kind of distributed software architecture to operate a trusted, unchangeable ledger. Parties can execute and store any kind of peer-to-peer transactions over the internet without the need of a trusted intermediary party. However, the Bitcoin blockchain is unlikely to meet the requirements for all usage scenarios (Xu et al. 2017). This is why various other implementations and combinations of the technology emerged for different requirements. Blockchain is a new technology and the accepted terminology is still evolving. In this paper, we use the term distributed ledger technology (DLT) as an overarching term (Hileman and Rauchs 2017) that can capture all potential design options.

Application of DLT's has been structured into three categories: Blockchain 1.0, 2.0, and 3.0 (Swan 2015). Blockchain 1.0 includes the initial use case of blockchain as a currency. Blockchain 2.0 refers to all applications using transactions combined with smart contracts for business related cases. Shermin (2017) points out that DLT's have the potential to become the new economic layer on top of the internet. From a business perspective, DLT is likely to impact finance and accounting, sales and marketing, legal affairs, and raising capital (Tapscott and Tapscott 2016). Therefore, DLT affects the main institutional pillars of industries and potentially disrupts business models by authenticating traded goods, improving operational efficiency, and facilitating disintermediation (Nowiński and Kozma 2017). This is in line with the main advantages for business processes of DLT showed by Viryasitavat et. al. (2018), namely enabling trust, faster transactions, and cost reductions through disintermediation. In conclusion, most literature agrees that DLT should not be neglected when looking at future business development. On top, blockchain 3.0 includes applications beyond finance, economics and markets, particularly in the areas of government, health, science, literature, culture, and art (Swan 2015). Finally, London et. Al. (2018) stated that apart from the purist or academic value coming from DLT's, they can also be used as a banner to modernize an industry from a digitization and collaboration perspective. DLT is gaining traction around the world in different industries. The report of
Digital Catapult (2018) and Hileman and Rauchs (2017) give empirical evidence for the UK and global market respectively.

\section{Technology Stack}

The goal is to identify factors influencing the DLT design decision by focusing on the most important aspects. Detailed explanations on the underlying base technologies for DLT's can be found for example in Wattenhofer (2017). Furthermore, various scholarship (Ballandies et al. 2018; Tasca and Tessone 2017; Xu et al. 2017) introduce taxonomies for DLT's and provide detailed information on different components. They were used as sources for this section. The information was structured based on an adapted version of the technology stack (Shermin 2017), pictured in Figure 1. The internet acts as the base technology for information sharing. A DLT, sometimes also referred to as protocol layer (Hileman and Rauchs 2017), is built on top of the internet layer with three main components impacting its characteristics: Ledger, P2P Network, and Governance. An application layer is possible, if code can be executed on the protocol layer.

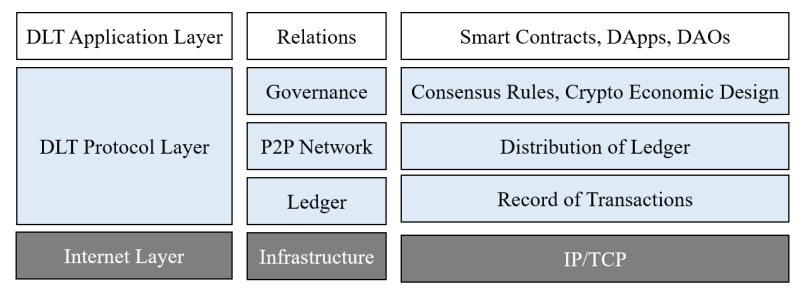

Figure 1: Technology stack (adapted from Shermin 2017)

\section{- Ledger:}

The ledger represents the data structure of the DLT and can be, as in the case of Bitcoin, a blockchain with sequential entries and total order (Ballandies et al. 2018). Another type of ledger is the directed acyclic graph (DAG) with a stream of individual transactions entangled together (e.g. IOTA, see iota.org). Various elements of a ledger can be defined, such as the storage capabilities or data encryption, which is beyond the scope of this paper. Most importantly, if the ledger supports turing complete language on the protocol layer, an application layer for coded relations is possible (see Figure 1). This enables the use of smart contracts, described the first time by Szabo (1996). These self-executing and unchangeable codes open up a large scope of applications. They allow for automated and self-executing conditions based on the state of the ledger. Many smart contracts can be combined to build so-called decentralized applications (DApps) or decentralized autonomous organizations (DAOs), also described in the analysis part of this paper. Furthermore, so-called tokens for all kind of (mostly financial) purposes can be introduced (see Token Alliance 2018).

\section{- P2P Network:}

The ledgers are distributed on different nodes in the network. These nodes can be either permissionless, meaning everyone could set up a node and write transactions to the ledger, or permissioned, by limiting 
write-access to the ledger. The second distinction is between public, meaning everyone can read the ledger, or private, where only defined members can access transactions on the ledger. The distribution and ownership of nodes impacts the decentralization of the system. Public permissionless DLT's naturally lead to higher decentralization, as everyone can set up a node, leading to more nodes and higher variability in interests of the participating users.

\section{- Governance:}

The governance of the DLT defines the set of rules for users interacting with the system. The most important component is the consensus mechanism, responsible for defining how to write, validate, and agree on entries to the ledger. Next to proof-of-work, which was the real innovation behind Bitcoin (see Nakamoto 2008), also other consensus mechanism have been introduced such as proof-of-stake (see e.g. Tasca and Tessone 2017). A consensus mechanism for a public DLT is enabled by a crypto-economic design (CED). A native coin of the DLT incentivizes participants to behave in the interest of the system. This is important to prevent attacks, but also to compensate miners for their expenditures in running nodes and adding transactions. Multiple properties of a CED can be defined, influencing the DLT's governance (see also Ballandies et al. 2018). A private DLT might not necessarily need a CED, as consensus is often based on permissions. This can have an impact on the cost structure for users when interacting with different systems. Often, users pay for transactions on a public DLT with transaction fees in its native token. In contrary, for private DLT's costs for the acquisition and maintenance of the infrastructure are predominant, while making transactions involves mostly no fee.

\section{Fundamental Properties}

The fundamental properties of DLTs are immutability, non-repudiation, integrity, and transparency $(\mathrm{Xu}$ et al. 2017). If the network is decentralized and protected through a working consensus-mechanism, the chain is immutable. Each transaction is added only once, which leads to non-repudiation of the stored data. The cryptographic tools support data integrity, allowing to verify that all the data is complete and as initially written into the ledger. Public access for everyone ensures transparency, and equal rights allow every participant the same ability to access and write to the ledger. Public permissionless DLT's are able to achieve the highest level of trust. Trust in the DLT is achieved because the participants rely on the fundamental properties of a DLT itself rather than on trusted third-parties. The more permissions, the less trust in the technical system can be accomplished. Even though integrity can also be maintained through cryptography, permissions have an impact on the other aspects of the fundamental properties. In private DLT's, immutability and non-repudiation are often ensured through verifying nodes or permissionbased consensus algorithms, with lower decentralization and increased possibility to act in self-interested behavior. Moreover, restricting read access to the ledger decreases transparency. This lowered trust in the technical system needs to be compensated by trusting the involved parties or a third party. Table 1 adapted from Xu et. Al. (2017) summarizes this for central databases and three typical design decisions of DLT's: private, public permissioned, and public permissionless. The fundamental properties are less favorable with more permissions. Having said that, in some use cases this high trust in the technical system might not be needed. A more centralized system offers a better performance (scalability and latency), as fewer nodes are involved and it does not need to continuously reach consensus with resource intensive algorithms. In addition, privacy can be of concern with public DLT's, if data encryption is considered too weak of a protection or parties want to have the possibility to control more aspects of the DLT (e.g. for easier implementation of system changes).

\section{Opportunities for DLT in Construction}

One vision for a digitized future construction process is described by some scholars as a combination of DLT, BIM, and the Internet of Things (IoT). IoT describes an environment where physical objects connect with the digital world, having sensors and connected devices

Table 1: Impact on DLT-related design decisions: fundamental properties and performance, using a spectrum from low (+) to high $(++++)$ (adapted from Xu et al. 2017).

\begin{tabular}{ccccc}
\hline Design Decision & Comment & Examples $^{l}$ & $\begin{array}{c}\text { Fundamental } \\
\text { Properties }\end{array}$ & Performance \\
\hline Fully Centralized & $\begin{array}{c}\text { Central databases with a single or } \\
\text { alternative providers }\end{array}$ & - & + & +++ \\
\hline Private DLT & $\begin{array}{c}\text { Private DLT's with permissions on } \\
\text { both read \& write of transactions }\end{array}$ & $\begin{array}{c}\text { Hyperledger } \\
\text { Fabric / Corda }\end{array}$ & ++ & ++ \\
\hline $\begin{array}{c}\text { Public Permissioned } \\
\text { DLT }\end{array}$ & $\begin{array}{c}\text { Public DLT's (permissionless read } \\
\text { access) \& permissions for write-access }\end{array}$ & IOTA & ++ & ++ \\
\hline $\begin{array}{c}\text { Public } \\
\text { Permissionless DLT }\end{array}$ & $\begin{array}{c}\text { Public DLT's (permissionless read } \\
\text { access) \& permissionless write-access }\end{array}$ & Ethereum & ++++ & + \\
\hline
\end{tabular}

${ }^{1}$ Examples classified by Ballandies et. al. (2018): Ethereum (www.ethereum.org), IOTA (www.iota.org), Hyperledger Fabric (www.hyperledger.org/projects/fabric), and Corda (www.r3.com). 
allowing to monitor and sense the environment with high spatial and time resolution (Fleisch 2010). Ye et al. (2018) describe in their conference paper a so-called "cup of water theory." They compare the technological environment with the analogy of a water glass and state that DLT will be the "cup wall," which is responsible to hold the data produced by IoT in a transparent, secure and convenient environment. The "cup-bottom" and base for the new technologies is BIM as the main tool to digitize construction project data. The combination of the three would link the digital models to the physical buildings, enabling real-time digital twins to automate procedures. Additional literature implicitly aligns with this viewpoint. Mathews et al. (2017) list the required ingredients for a DLT adoption in the construction sector. Main ingredients include BIM and IoT. Additional consultancy reports (Kinnaird and Geipel 2017; Penzes 2018) concur that a future industry state will be characterized by the "circular economy of BIM things." The produced data from projects and IoT can be fed into a common data environment managed and visualized through BIM, enabling a digital twin over the whole life cycle of a building. Benefits of these digital twins exist for the planning and construction process as well as in the operational phase (Penzes 2018). Having a digital model from the very beginning in the project throughout the construction phase can reduce efforts for the operation of the facility over its lifecycle. As more activities become digitalized, DLT's offer a way for business process optimization and automation on the transaction level through the use of smart contracts for higher efficiency and accuracy. Furthermore, DLT could benefit construction with higher transparency in the project and supply chain due to data immutability and traceability.

\section{Challenges Facing DLT in Construction}

Despite the potential benefits of DLT in construction, many challenges exist for its successful application and adoption. Apart from technical barriers of DLT, various challenges regarding the nature of the construction industry and the involved humans exist, among them lack of awareness, skills, as well as resistance to change (Wang et al. 2017). Some scholars see the coding of relations into smart contracts as an especially challenging area in construction. Mason (2017) lists construction contracts of all kinds and investigates if a transition to smart contracts would be possible. He notes that smart contracts work best where they are short-term or are of instantaneous effect. This is sometimes at odds with the complicated and longrunning nature of construction projects. He believes that certain aspects of the construction contract cannot be fully intelligent, and the best that can be achieved in the short to medium term is a semi-automated position with human involvement. He found in a survey that construction industry participants mostly doubt that full automation with smart contracts is possible (Mason and Escott 2018). Furthermore, Kinnaird and Geipel (2017) emphasize that transaction entries in the DLT do not verify the correctness of data itself, therefore moderators might need to be involved to resolve disputes related to data sources. Belle (2017) summarized this saying that two kind of transactions are difficult for smart contracts: 1) ambiguous situations where third party expertise is needed; and 2) when turning a task, negotiation, or contract terms into algorithms is difficult. That is why some authors (e.g. Kinnaird and Geipel 2017; Mathews et al. 2017) propose to look more into oracles, which are third party or external information sources that interact with a smart contract. This can be human interaction, data from sensors or thirdparty services. Oracles might offer a solution to deal with problems of wrong or ambiguous information and implementation. These challenges has been summarized using three dimensions: technical, political, and social in Li et al. (2018).

As DLT has developed, academics and consultants have theorized the potential use cases for the technology. These use cases are a helpful starting place to understand how DLT can benefit the construction industry. However, there is an emerging gap between the described use case ideas and the technical system implementation required for DLT (Ye et al. 2018). To solve the emerging challenges described above, applications of DLT in construction must take the appropriate DLT design options into consideration. Yet no literature was found investigating what technical design option might be best suited for which use case in construction. Turk and Klinc (2017) started to think about a possible technical layout for the connection of blockchain and BIM. However, the proposed scenarios do not distinguish between different DLT's. The question remains: how does one know which DLT to design for a certain use case? Furthermore, how can these use cases be clustered into broader categories based on their technical requirements for DLT?

\section{Methodology}

To answer these two questions, this paper conducts a review of recent papers written about DLT use cases. Because literature on DLT in construction is still limited, both scholarship and consulting reports were considered. The literature review conducted by Li et al. (2018) was used as a starting point to identify relevant papers. We then refined the papers reviewed based on relevance to the construction industry, and limited the literature to papers published in 2017 or later. In total, we identify nine papers written about DLT use cases (see Table 2).

$$
\begin{gathered}
\text { Table 2: Literature for use-case analysis (S: scholarly papers, } \\
\text { C: consulting reports) }
\end{gathered}
$$

\begin{tabular}{ccc}
$\#$ & Literature & Type \\
\hline$[1]$ & (Belle 2017) & $\mathrm{S}$ \\
{$[2]$} & (Heiskanen 2017) & $\mathrm{S}$ \\
{$[3]$} & (Kinnaird and Geipel 2017) & $\mathrm{C}$ \\
{$[4]$} & (Mason 2017) & $\mathrm{S}$ \\
{$[5]$} & (Mathews et al. 2017) & $\mathrm{S}$ \\
{$[6]$} & (Penzes 2018) & $\mathrm{C}$ \\
{$[7]$} & (Turk and Klinc 2017) & $\mathrm{S}$ \\
{$[8]$} & (Wang et al. 2017) & $\mathrm{S}$ \\
{$[9]$} & (Ye et al. 2018) & $\mathrm{S}$
\end{tabular}

From these nine papers, we identify 22 individual use cases (see Table 3). Afterwards, use-case categories were 
identified based on the clustering of use cases regarding similarities in their user group and/or their higher level application. If no use case was left without a category, it implied that they were able to capture the current state of DLT applications in construction.

In a second step, the categories were evaluated according to their technical requirements based on the characteristics of the respective user group. If the requirements of one category varied from the other categories, each category was needed. The assessment from both the use case and technical view point leads to a robust argument that the proposed use-case categories are eligible for a first assessment regarding their technical design decisions.

In the end, we identify six use-case categories. For each category, we describe the overarching characteristics identified in the literature, as well as the specific use case applications. Finally, we propose a basic framework and analysis to identify which DLT design is best suited for each use-case category.

\section{Findings}

Six final use-case categories were identified (Table 3):

1. Internal Automation of Administrative Processes

According to Wang et. Al. (2017), DLT could be used for storage and perfect notarization of each creation, deletion, and updating of files across an inter-organizational system (Table 3, 1.1). This can simplify and automate administrative processes. Wang et. Al. (2017) mentions the recording of quality data or resource consumption data as examples.

2. Transaction Automation \& Optimization Between Stakeholders

This use-case category focuses on the automation of transactions with smart contracts between different stakeholders, instead of one stakeholder as in the first use case category. The most mentioned use case in this category was the automatic triggering of payments (Table $3,2.1$ ), as delays for monetary transactions have been mentioned repeatedly as a factor causing conflicts and disputes (Eastman 2011). This can be generalized to trigger various deliverables from contracts (Table 3, 2.2). Once a smart contract is written, its behavior is unambiguous and predictable. This can be used for self- executing contract administration (Table 3, 2.3), such as monitoring and updating of the contract status (Wang et al. 2017). Smart contracts are also mentioned as a way to enable automated information and data sharing in projects (Table 3, 2.4), ensuring consistent reporting for (sub)contractors and owners. Combined with digital signatures (Table 3, 2.5), decisions can be quickly approved and automatically trigger subsequent action. These use cases are independent of the project phase and can be applied for procurement and supply chain activities for higher accuracy and efficiency.

\section{Record of Transactions, Changes, Ownership}

The focus lies here on the immutability and transparency a DLT can provide by timestamping value transactions (Table 3, 3.1). The most mentioned use case is the record of changes and ownership (Intellectual Property) of digital models and components, especially in combination with BIM (Table 3, 3.2). Moreover, the ownership of physical property can be registered on a DLT (Table 3, 3.2). One other often mentioned use case is the tracking of supply chain logistics, including procurement, transportation, and storage of goods (Table 3, 3.4). Penzes (2018) expanded this towards use cases such as the tracking of project progress and worked hours (Table 3, 3.5), maintenance and operations data of buildings (Table 3, 3.6), health \& safety incidents (Table 3, 3.7), and machine operations data (Table 3, 3.8).

\section{Decentralized Applications (DApps)}

DApps enable direct user interaction with a DLT, typically through web user interfaces. Even though it is possible to create web applications for the use cases in the previous categories during a project, we refer with DApps in this category to long-term and global users. They might be unknown and involved in various projects simultaneously. Different use cases for DApps are mentioned, such as managing identities (ID's) of people or organizations on a DLT (Table 3, 4.1) for clear and trustworthy identification and reputation (see e.g. civic.com as an existing DApp for managing ID's). Similarly, material and product passports with provenance-related information (Table 3, 4.2) can be maintained throughout the supply chain. This can be used for quality assurance in global construction projects (Wang et al. 2017) or to enable the reuse of materials at a later stage of a building towards a circular economy

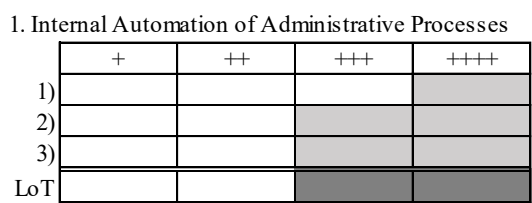

4. Decentralized Applications (DApps)

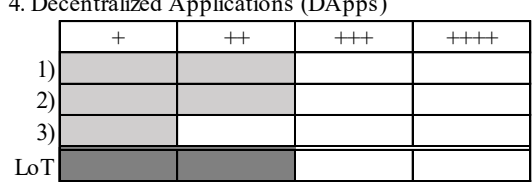



5. Decentralized Autonomous Organizations (DAOs)

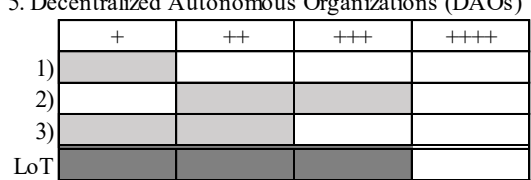

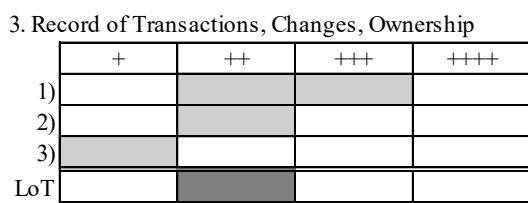

6. Coins/Tokens as Payment or Incentive Scheme

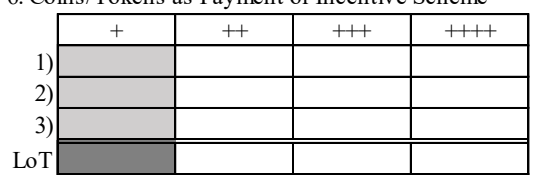

Figure 2: Assessment for level of Trust (LoT) from low (+) to high (++++). 1) Are users unknown (+) or known (++++)? 2) Do users have opposite (+) or aligned (++++) interests? 3) Is the data used long-term (+) or short-term (++++)? 
Table 3: Use-Case clustering into six categories, based on the literature listed in Table 2.

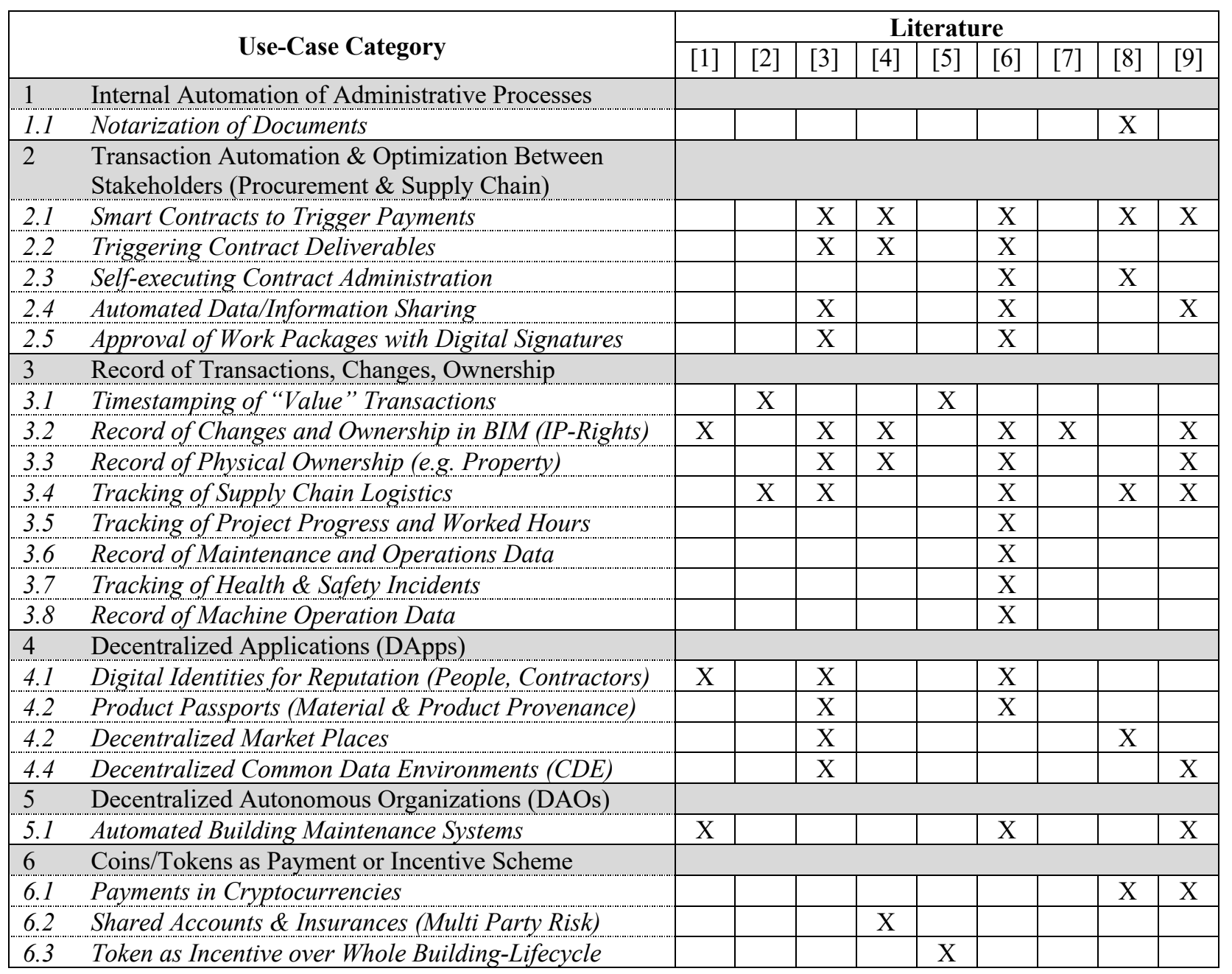

(Kinnaird and Geipel 2017). Also, certification of products and buildings can profit from the availability of this trusted data. Decentralized marketplaces (Table 3, 4.3) can be set up based on the ID's for objective data of the bestqualified person or company in tendering, without the need to disclose sensitive data to third parties (Belle 2017). Lastly, decentralized common data environments (CDE) as a combination of cloud storage and DLT (see e.g. sia.tech or storj.io) are proposed to store digital models without the need to trust a third party server provider or run private servers vulnerable to attacks (Ye et al. 2018).

5. Decentralized Autonomous Organizations (DAOs)

DAOs represent a fully autonomous organization based on smart contracts, only running on a DLT without any human involvement. Governance rules are coded in smart contracts and incentive mechanisms are implied through crypto-economic design (CED). Even though fully automated construction companies seem futuristic, three sources (Belle 2017; Penzes 2018; Ye et al. 2018) mentioned automated building maintenance systems as one possibility for a DAO (Table $3,5.1$ ). The idea is that building performance can be monitored through sensors (IoT) in combination with BIM, enabling an automatized reaction to certain conditions based on predefined rules. This can include automatic ordering of spare parts or regulating technical installations.

6. Coins/Tokens as Payment or Incentive Scheme

This last use-case category includes all financial and incentive related use cases based on DLT's. An obvious use case is the payment in cryptocurrencies (Table 3, 6.1), which allows to send money across boarders instantly and with small fees. Shared risk and reward structures with shared bank accounts and insurances could be further application (Table 3, 6.2). Finally, Mathews et al. (2017) propose the use of an \#AECoin as a token to reward project contributors for the value of an artefact over its full life cycle. This should create superior value for the project, as contributors are incentivized to work better in order to earn from the value creation after the project handover to the client.

\section{Framework for Technical Design Decisions}

Having identified the categories based on use-case clustering (Table 3), we analyze them regarding their technical requirements. As seen in Table $1, \mathrm{Xu}$ et al. (2017) structures different DLT design options according 
Table 4: Identification of suited DLT design options for each use-case category. Range of "level of trust" (from Figure 2) and "fundamental properties" from low (+) to high (++++).

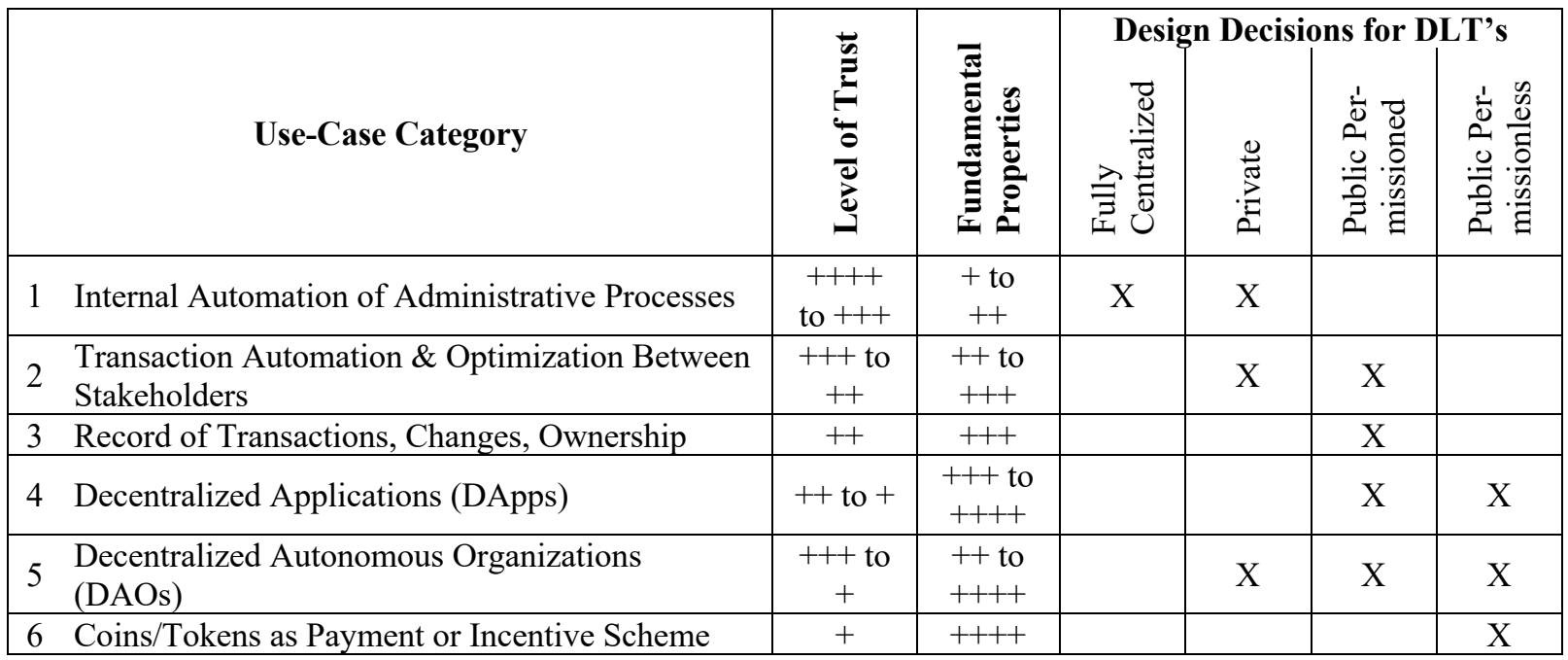

to their fundamental properties. With favorable fundamental properties, trust in the system is established, which is needed for use cases with low trust. Therefore, we introduce the needed level of trust for a use-case category as an inversely related proxy to the fundamental properties of a DLT. The level of trust was quantified for each category in Figure 2 based on three questions, which relate to two different kinds of trust identified. 1) Are the users known or unknown?, and 2) Do they have aligned or opposite interests?, relate to the participants behavior and relationship in a use case. Unknown users and unaligned interests lead to less trust. Question 3) Is the data used long-term or short-term?, relates to the required trust and transparency in the data. Data used long-term across project boundaries or over the whole life cycle of a building requires more trust. The final proxy level of trust was assigned based on an approximate average of the three questions from low $(+)$ to high $(++++)$. As the level of trust is inversely related to the fundamental properties of a DLT, we derive the needed fundamental properties of the specific use-case category as the opposite of the level of trust (e.g. the level of trust is high $(++++)$, then the required fundamental properties are low $(+))$. Using the relationships introduced in Table 1 , this results in one or more of the four introduced design decisions for each usecase category. The analysis is shown in Table 4 and discussed in the following section.

\section{Discussion}

The qualitative assessment of trust is not always clearly assignable and the assessment was based on the author's evaluation. The first category involves single organizations or few known stakeholders. Trust is therefore rather high and a DLT-solution not necessarily needed. A cloud service or versioning servers such as git (git-scm.com) could be alternatives. Having said that, a DLT could enable later integration with other stakeholders (second use-case category) or might make sense if interests internally are not aligned. The second category involves multiple stakeholders with different interests.
However, these stakeholders normally work on the same project and know one another. Data is mostly needed during the project and does not cross project boundaries. Dependent on the specific use case, both a public or private ledger could be suited. In contrast, use cases in the third category are more likely to store data long-term for transparency and accountability reasons. Furthermore, the network can be more global and complex as e.g. in supply chain tracking, and participants are often unknown. At least a public permissioned DLT is desirable. Having said that, one should be careful if the record is relevant for conflicting parties. E.g. for use cases in Table 3, 3.5-3.8, if this data is only relevant for one party, a private DLT or even centralized database might be sufficient (see also first use-case category). DApps (fourth use-case category) involve long-term and global users that do not know each other across project boundaries with various interests. A public DLT is for this case favorable. For DAOs (fifth usecase category), the case is more complex. A DAO for building maintenance could be only for one particular building, still owned by a company. For such cases they could be set up on private DLT's. If they are autonomous, representing their own organizational entity, a public DLT makes sense. The payment or incentive use cases in the sixth category require high trust in the technical system, preferably with a public permissionless DLT.

The proposed six use-case categories capture the current state of proposed use cases in the construction industry (Table 3). Furthermore, they differ in terms of prerequisites for DLT design, because the respective user group varies in terms of level of trust (Figure 2 and Table 4). This leads to a robust assessment that these use-case categories are complete and the proposed framework is suited to identify DLT design options for construction use cases.

However, we note the findings presented above should be considered with the following limitations:

- The literature of DLT in construction is still limited 
and the categories cover the current state of knowledge. Hence, use cases might evolve in the coming years and the categories should be revised.

- The framework is sensitive towards the qualitative proxy level of trust. With a different viewpoint on the user group, this might lead to other assessments of suited DLT design options than in Table 4.

- The nature of the proxy level of trust generates results found in a certain range rather than an absolute value. This leaves the decision makers with the freedom to adjust the final DLT design solution regarding other factors.

- As the framework is only based on one proxy, other important factors such as scalability, privacy, and the cost structure are left out. Major research efforts are currently put into the advancement of DLT in technical regards, and scalability and privacy implications between DLT design choices might become less decisive in the future. Nevertheless, a more detailed framework with additional factors could be a topic of future research.

- The selection of design options is limited to four cases (see Table 1). This can be of course refined, also considering combinations between several design options (hybrid-solutions).

Despite above limitations, the proposed use-case categories together with this basic framework can lead to a more connected and structured thinking between technological properties of DLT's and use cases in construction. They allow a first assessment of DLT design options based on a simple proxy: the level of trust of the user group. It is important to consider technical aspects of DLT's when thinking about use cases. With this, the authors hope to contribute towards more case-studies of DLT in a construction context to further advance the research in this field. Even with our basic assessment, different requirements for DLT in construction are identified. This might imply that a future connected ecosystem of DLT's could be preferable over a one-sizefits-all DLT solution for construction.

Finally, based on the literature review of both DLT and its construction-related applications, the following future research areas were identified:

- An extended framework beyond the introduced metric level of trust to connect DLT use cases to the best suited DLT design option.

- Interoperability of different DLT's together with existing software solutions in construction.

- Establishing DLT standards for the specific needs of the construction industry.

- Best practices for coding construction applications into smart contracts and the role of oracles.

- Quantitative assessment of use-case feasibility through case-studies.

- More general impact of DLT on the construction industry ecosystem in a socio-economic context.

\section{Conclusion}

This paper reviews literature regarding DLT and its applications for construction. It was found that the benefits of DLT theoretically align with some of the trust problems facing the construction industry. The overall research is still limited and various challenges for implementation remain. Furthermore, there is no evidence for the socioeconomic benefit of DLT's in construction yet. Especially, case-studies of use cases in construction are missing. Therefore, this paper proposed and described six use-case categories through use-case clustering. The paper then provides a basic framework to link use-case categories to possible design decisions of DLT's based on the trust-level in the user group. The resulting framework can be used by practitioners and academics to reduce the gap between DLT use-case ideas and technical system implementation.

\section{References}

Agarwal, R., Chandrasekaran, S., and Sridhar, M. (2016). Imagining construction's digital future. McKinsey \& Company.

Ballandies, M. C., Dapp, M. M., and Pournaras, E. (2018). Decrypting Distributed Ledger Design - Taxonomy, Classification and Blockchain Community Evaluation.

Belle, I. (2017). The architecture, engineering and construction industry and blockchain technology. JI, G. \& TONG, Z. (eds.) Digital Culture 数码文化 Proceedings of the 2017 National Conference on Digital Technologies in Architectural Education, Nanjing, China Architecture Industry Publishers.

Digital Catapult. (2018). Blockchain in Action. https://www.digicatapult.org.uk/news-andviews/publication/blockchain-in-action-state-ofthe-uk-market/ (Dec. 15, 2018).

Dubois, A., and Gadde, L.-E. (2002). The construction industry as a loosely coupled system: implications for productivity and innovation. Construction Management and Economics, 20(7), 621-631.

Fleisch, E. (2010). What is the Internet of Things ? - An Economic Perspective. Auto-ID Labs White Paper WP-BIZAPP-053, 5(2), 1-27.

Hall, D., Algiers, A., Lehtinen, T., Levitt, R. E., Li, C., and Padachuri, P. (2014). The Role of Integrated Project Delivery Elements in Adoption of Integral Innovations. Proceedings of the Engineering Project Organization Conference 2014, Denver, P. Chan and R. Leicht, eds., Engineering Project Organization Society, Devil's Thumb Ranch, Colorado, 1-20.

Hall, D. M., and Scott, W. R. (2019). Early Stages in the Institutionalization of Integrated Project Delivery. Project Management Journal, 50(2), 875697281881991.

Heiskanen, A. (2017). The technology of trust: How the 
Internet of Things and blockchain could usher in a new era of construction productivity. Construction Research and Innovation, Taylor \& Francis, 8(2), 66-70.

Hileman, G., and Rauchs, M. (2017). 2017 Global Blockchain Benchmarking Study. SSRN Electronic Journal.

Kinnaird, C., and Geipel, M. (2017). Blockchain Technology: How the Inventions Behind Bitcoin are Enabling a Network of Trust for the Built Environment. Arup.

Li, J., Greenwood, D., and Kassem, M. (2018). Blockchain in the built environment: analysing current applications and developing an emergent framework. Proceedings of the Creative Construction Conference 2018, Ljubljana, 1-10.

London, S., Carson, B., and Higginson Matt. (2018). Blockchain explained: What it is and isn't, and why it matters. McKinsey \& Company, https://www.mckinsey.com/business-

functions/digital-mckinsey/our-insights/blockchainexplained-what-it-is-and-isnt-and-why-it-matters

(Oct. 3, 2018).

Mason, J. (2017). Intelligent Contracts and the Construction Industry. Journal of Legal Affairs and Dispute Resolution in Engineering and Construction, 9(3), 04517012.

Mason, J., and Escott, H. (2018). Smart contracts in construction: Views and perceptions of stakeholders. Proceedings of the FIG Conference, Istanbul.

Mathews, M., Robles, D., and Bowe, B. (2017). BIM+Blockchain: A Solution to the Trust Problem in Collaboration? CITA BIM Gathering.

Miettinen, R., and Paavola, S. (2014). Beyond the BIM utopia: Approaches to the development and implementation of building information modeling. Automation in Construction, Elsevier B.V., 43, 8491.

Nakamoto, S. (2008). Bitcoin: A Peer-to-Peer Electronic Cash System. www. bitcoin.org.

Nowiński, W., and Kozma, M. (2017). How Can Blockchain Technology Disrupt the Existing Business Models? Entrepreneurial Business and Economics Review, 5(3), 173-188.

Papadonikolaki, E. (2018). Loosely Coupled Systems of Innovation: Aligning BIM Adoption with Implementation in Dutch Construction. Journal of Management in Engineering, American Society of Civil Engineers, 34(6), 05018009.

Papadonikolaki, E., and Wamelink, H. (2017). Inter- and intra-organizational conditions for supply chain integration with BIM. Building Research \& Information, Routledge, 45(6), 649-664.
Penzes, B. (2018). Blockchain technology: could it revolutionise construction? Institution of Civil Engineers.

Pishdad-Bozorgi, P., and Beliveau, Y. J. (2016). Symbiotic Relationships between Integrated Project Delivery (IPD) and Trust. International Journal of Construction Education and Research, 12(3), 179192.

Shermin, V. (2017). Disrupting governance with blockchains and smart contracts. Strategic Change, Wiley-Blackwell, 26(5), 499-509.

Swan, M. (2015). Blockchain. O’Reilly Media.

Szabo, N. (1996). Smart Contracts: Building Blocks for Digital Markets. http://www.fon.hum.uva.nl/rob/Courses/Informatio nInSpeech/CDROM/Literature/LOTwinterschool20 06/szabo.best.vwh.net/smart_contracts_2.html (Nov. 23, 2018).

Tapscott, D., and Tapscott, A. (2016). How Blockchain Will Change Organizations. MIT Sloan Management Review. 58(2), 10-13.

Tasca, P., and Tessone, C. J. (2017). Taxonomy of Blockchain Technologies. Principles of Identification and Classification.

Thomsen, C., Darrington, J., Dunne, D., and Lichtig, W. (2009). Managing Integrated Project Delivery. Construction Management Association of America, McLean, Virginia, USA.

Token Alliance. (2018). Understanding Digital Tokens: Market Overviews and Proposed Guidelines for Policymakers and Practitioners. Token Alliance Whitepaper, Chamber of Digital Commerce.

Turk, Ž., and Klinc, R. (2017). Potentials of Blockchain Technology for Construction Management. Procedia Engineering, Elsevier, 196, 638-645.

Viryasitavat, W., Da Xu, L., Bi, Z., and Sapsomboon, A. (2018). Blockchain-based business process management (BPM) framework for service composition in industry 4.0. Journal of Intelligent Manufacturing, Springer US, 1-12.

Wang, J., Wu, P., Wang, X., and Shou, W. (2017). The outlook of blockchain technology for construction engineering management. Frontiers of Engineering Management, 4(1), 67.

Wattenhofer, R. (2017). Distributed Ledger Technology: The Science of the Blockchain (2nd ed.). CreateSpace Independent Publishing Platform, USA.

Whyte, J. K., and Hartmann, T. (2017). How digitizing building information transforms the built environment. Building Research and Information, Taylor \& Francis, 45(6), 591-595.

Xu, X., Weber, I., Staples, M., Zhu, L., Bosch, J., Bass, L., 
Pautasso, C., and Rimba, P. (2017). A Taxonomy of Blockchain-Based Systems for Architecture Design. Proceedings of the 2017 IEEE International Conference on Software Architecture (ICSA), Gothenburg, IEEE, 243-252.

Ye, Z., Yin, M., Tang, L., and Jiang, H. (2018). Cup-ofWater theory: A review on the interaction of BIM, IoT and blockchain during the whole building lifecycle. (Isarc).

Zolin, R., Hinds, P. J., Fruchter, R., and Levitt, R. E. (2004). Interpersonal trust in cross-functional, geographically distributed work: A longitudinal study. Information and Organization, 14(1), 1-26. 